\title{
New Treatment Options for the Management of Multiple Myeloma
}

\author{
Presented by Shaji K. Kumar, MD
}

\begin{abstract}
Multiple myeloma (MM) is a complex disease characterized by considerable genetic heterogeneity. The updated NCCN Guidelines for MM have added new "preferred" regimens for transplant and nontransplant candidates, and have moved some formerly "preferred" regimens to the "other" category. Supportive care has improved outcomes for patients, and new treatments in combination have extended survival for patients with MM. Novel agents on the horizon are poised to raise the bar even further.
\end{abstract}

J Natl Compr Canc Netw 2017;15(5.5):709-712

Shaji K. Kumar, MD, Professor of Medicine, Mayo Clinic Cancer Center, reviewed the significant changes in the therapeutic options and understanding of the biology of multiple myeloma (MM) at the NCCN 22nd Annual Conference.

"The first step in approaching MM is to make sure the diagnosis is correct. Identifying the time point for therapy is critical. Despite effective treatment, most patients will eventually relapse. Hopefully we can offer patients an extended, treatment-free interval," Dr. Kumar said.

The 2017 NCCN Clinical Practice Guidelines in Oncology (NCCN Guidelines) for MM incorporates an expanded definition of the diagnosis of active MM that includes the introduction of several biomarkers of active disease. The old definition relied on hypercalcemia, renal insufficiency, anemia, and bone disease (CRAB), whereas the expanded definition now includes bone marrow plasma cell involvement $\geq 60 \%,>1$

\footnotetext{
Presented by Shaji K. Kumar, MD, Division of Hematology, Mayo Clinic Cancer Center, Rochester, Minnesota.

Dr. Kumar has disclosed that he receives grant/research support from Abbott Laboratories, Amgen Inc.; Celgene Corporation; Janssen Pharmaceutica Products, LP; Novartis Pharmaceuticals Corporation; sanofi-aventis U.S.; and Takeda Pharmaceuticals North America, Inc. He serves as a scientific advisor for Amgen Inc., Bristol-Myers Squibb Company, Celgene Corporation, GlycoMimetics, Janssen Pharmaceutica Products, LP, sanofi-aventis U.S., and Takeda Pharmaceuticals North America, Inc.; and has received consulting fees/honoraria from Kesios Therapeutics, NOXXON Pharma AG, and SkylineDx.

Correspondence: Shaji K. Kumar, MD, Division of Hematology, 200 First Street SW, Rochester, MN 55905. E-mail: kumar.shaji@mayo.edu
}

lesion on PET/MRI, and free light chain ratio $\geq 100$, in addition to CRAB.

The new version of the guidelines also includes risk stratification of myeloma. "MM is not one disease. Previously, the International Staging System [ISS] grouped patients into 3 stages for prognostication based only on serum $\beta-2$ microglobulin and serum albumin levels. More recently, the older staging system was expanded to include serum lactate dehydrogenase and high-risk genetic aberrations," he continued (Table 1). Additionally, initial diagnostic workup should now include metaphase cytogenetics on bone marrow and plasma cell fluorescence in situ hybridization to identify genetic abnormalities that include del13, del17p13, t $(4 ; 14)$, $\mathrm{t}(11 ; 14), \mathrm{t}(14 ; 16)$, and 1q21 amplification.

\section{Initial Therapy for Newly Diagnosed MM}

"The ideal therapy should rapidly and effectively control disease, reverse disease-related complications, decrease the risk of early death, be easily tolerated with minimal toxicity, and not interfere with stem cell collection for transplant," Dr. Kumar stated. The "tool box" of ideal therapies includes combinations of immunomodulatory drugs (IMiDs; thalidomide, lenalidomide), proteasome inhibitors (bortezomib, carfilzomib, ixazomib), traditional chemotherapy (cyclophosphamide, doxorubicin), and, more recently, monoclonal antibodies (daratumumab, elotuzumab). 


\begin{tabular}{|c|c|}
\hline Prognostic Factor & Criteria \\
\hline \multicolumn{2}{|l|}{ ISS stage } \\
\hline 1 & $\begin{array}{l}\text { Serum } \beta_{2} \text {-microglobulin }<3.5 \mathrm{mg} / \mathrm{L} \text {, serum } \\
\text { albumin } \geq 3.5 \mathrm{~g} / \mathrm{dL}\end{array}$ \\
\hline II & Not ISS stage I or III \\
\hline III & Serum $\beta_{2}$-microglobulin $\geq 5.5 \mathrm{mg} / \mathrm{L}$ \\
\hline \multicolumn{2}{|l|}{ CA by iFISH } \\
\hline High risk & $\begin{array}{l}\text { Presence of del }(17 p) \text { and/or translocation } \\
t(4 ; 14) \text { and/or translocation } t(14 ; 16)\end{array}$ \\
\hline Standard risk & No high-risk CA \\
\hline \multicolumn{2}{|l|}{ LDH } \\
\hline Normal & Serum LDH $<$ the upper limit of normal \\
\hline High & Serum LDB $>$ the upper limit of normal \\
\hline \multicolumn{2}{|c|}{ A new model for risk stratification for MM } \\
\hline \multicolumn{2}{|l|}{ R-ISS stage } \\
\hline 1 & $\begin{array}{l}\text { ISS stage I and standard-risk CA by iFISH } \\
\text { and normal LDH }\end{array}$ \\
\hline II & Not R-ISS stage I or III \\
\hline III & $\begin{array}{l}\text { ISS stage III and either high-risk CA by iFISH } \\
\text { or high LDH }\end{array}$ \\
\hline
\end{tabular}

Abbreviations: CA, chromosomal abnormalities; iFISH, interphase fluorescent in situ hybridization; ISS, International Staging System; $\mathrm{LDH}$, lactate dehydrogenase; MM, multiple myeloma; R-ISS, revised International Staging System.

From Palumbo A, Avet-Loiseau H, Oliva S, et al. J Clin Oncol 2015;33:2863-2869. Reprinted with permission. (22015 American Society of Clinical Oncology. All rights reserved.

\section{Transplant-Eligible Patients}

Transplant eligibility is based on patient age and comorbidities. In general, autologous stem cell transplant (SCT) is reserved for younger patients who are physically fit.

\section{Initial Therapy (Induction)}

The current standard of care for newly diagnosed patients is RVD (bortezomib/lenalidomide/dexamethasone). This recommendation is based primarily on data from the phase III SWOG S077 trial demonstrating that RVD improved progression-free survival (PFS) and overall survival (OS) compared with lenalidomide/dexamethasone in 525 patients with newly diagnosed MM. ${ }^{1}$

Other preferred regimens for primary therapy listed in the 2017 NCCN Guidelines include bortezomib/doxorubicin/dexamethasone and bortezomib/thalidomide/dexamethasone (both category 1). Other regimens that can be considered for primary therapy include bortezomib/dexamethasone (category 1), carfilzomib/lenalidomide/dexametha- sone, ixazomib/lenalidomide/dexamethasone, and lenalidomide/dexamethasone (category 1 ).

"In today's world, we have very good treatment options," he said. "But we are still trying to improve on these regimens. The new regimens achieve a good depth of response, better than what we have seen in the past in the absence of SCT."

The duration of maintenance therapy remains controversial. The phase III ENDURANCE trial is an ongoing trial comparing the standard of care (RVD) versus carfilzomib/lenalidomide/dexamethasone (CRD). Both arms are then followed by lenalidomide maintenance ( 24 cycles vs continuous maintenance). Results of this study should help clarify the optimal duration of maintenance therapy.

The addition of ixazomib to the regimen of lenalidomide and dexamethasone, as the proteasome inhibitor component, provides the first all-oral regimen, and studies of this drug are ongoing.

"The introduction of monoclonal antibodies [ie, daratumumab and elotuzumab] is the most exciting development in recent years," he noted. "Trials are planned or already ongoing that add daratumumab to the backbone regimens."

"The hope is that adding a monoclonal antibody to the triplet regimen can increase the depth of response, which can translate to improved survival, as well," he said. "But we cannot just assume that more is better." Clinical trials will provide the required evidence.

\section{Autologous SCT}

Once patients have achieved a response, autologous SCT is used to further deepen and maintain that response. Many studies show improved OS for autologous SCT, but in the modern era of more powerful therapies, experts have questioned whether autologous SCT is still necessary (for younger patients and those who are candidates for transplant).

A recent study examined whether autologous SCT is still necessary by comparing standard-of-care RVD followed by lenalidomide maintenance versus RVD followed by autologous SCT followed by lenalidomide maintenance. Results showed that the addition of autologous SCT improved depth of response, minimal residual disease (MRD)-negativity, and PFS when compared with no autologous SCT. To date, there has been no difference in OS between the arms. ${ }^{2}$ 
"This trial once again proves that transplant remains a standard of care for eligible patients. However, lack of a difference in OS suggests that transplant can be delayed until the time of relapse, if so desired," he stated.

\section{Consolidation and Maintenance Therapy}

Consolidation and maintenance therapy are thought to optimize responses achieved by primary therapy. A recent meta-analysis of 3 randomized clinical trials found that lenalidomide maintenance after autologous SCT significantly improved OS compared with controls. ${ }^{3}$ A subgroup analysis showed a benefit in most subgroups, with the exception being those with unfavorable cytogenetics and high-risk ISS stage.

"The downside of maintenance therapy with lenalidomide is an increase in second primary malignancies. It is important that patients on maintenance lenalidomide undergo routine, age-appropriate cancer screening," Dr. Kumar stated.

\section{Nontransplant-Eligible Patients}

The median age of patients diagnosed with MM is 69 years. A number of these patients may not be able to undergo autologous SCT.

The 2017 NCCN Guidelines list the following preferred regimens for nontransplant candidates: bortezomib/cyclophosphamide/dexamethasone, bortezomib/ lenalidomide/dexamethasone, and lenalidomide/dexamethasone. Other options for primary therapy in this patient population include bortezomib/dexamethasone, CRD, and ixazomib/lenalidomide/dexamethasone. Maintenance therapy regimens include bortezomib and lenalidomide. The recommendation for CRD carries a caveat that this regimen can be associated with cardiac and pulmonary toxicity, especially in older patients. Thus, the guidelines have added a footnote that states, "Triplet regimens should be used as the standard therapy for patients with MM; however, elderly or frail patients may be treated with doublet regimens."

The goal of therapy is to achieve a deep response while balancing toxicity, given the improved outcome in patients achieving MRD-negative status. "We still need to learn a lot about how to use MRD testing and results in the clinic," Dr. Kumar said. "Clinical trial designs are now incorporating MRD as a measurement, but it is not yet clear if MRD status should change therapy for current patients."

\section{Supportive Care/Adjunctive Treatment}

Supportive care measures have helped improve outcomes for patients with MM. The 2017 NCCN Guidelines recommend the following adjunctive treatment:

- Bisphosphonates for bone disease (with prior dental examination): monitor renal function and for osteonecrosis of the jaw.

- Hypercalcemia: treat with hydration, bisphosphonates, steroids, and/or calcitonin.

- Hyperviscosity: plasmapheresis should be used as adjunctive therapy for symptomatic hyperviscosity.

- Anemia: see the NCCN Guidelines for Cancerand Chemotherapy-Induced Anemia (available at NCCN.org); consider erythropoietin.

- Infection: see NCCN Guidelines for Prevention and Treatment of Cancer-Related Infections (available at NCCN.org); intravenous immunoglobulin should be considered in the setting of life-threatening infection. Consider pneumococcal and influenza vaccines.

- Antifungal prophylaxis should be considered if high-dose dexamethasone is part of the regimen. Herpes zoster prophylaxis for patients treated with a proteasome inhibitor.

- Renal dysfunction: maintain hydration; avoid use of nonsteroidal anti-inflammatory drugs. Plasmapheresis is a category $2 \mathrm{~B}$ recommendation; not a contraindication to transplant. Monitor for renal dysfunction with chronic use of bisphosphonates.

- Coagulation/thrombosis: full-dose aspirin recommended with immunomodulatory-based therapy; therapeutic anticoagulation recommended for those at high risk of thrombosis (see NCCN Guidelines for Cancer-Associated Venous Thromboembolic Disease).

\section{Relapsed Myeloma}

"The ideal approach is to treat the disease to its maximum response and maintain the patient on one drug until disease progression or lack of tolerability," Dr. Kumar said. Despite state-of-the-art treatment with powerful new drugs, most patients will eventually experience relapse.

Treatment options for patients with relapsed disease include newer classes of older drugs (IMiDs, proteasome inhibitors, monoclonal anti- 
bodies, histone deacetylase inhibitors); older agents and conventional chemotherapy (dexamethasone/ prednisone, cyclophosphamide, melphalan, anthracyclines); combinations of new and older drugs; a second autologous SCT; and enrolling patients on clinical trials of promising new drugs (ie, selinexor, venetoclax, checkpoint inhibitors, and chimeric antigen receptor T-cell therapy).

\section{References}

1. Durie BG, Hoering A, Abidi MH, et al. Bortezomib with lenalidomide and dexamethasone versus lenalidomide and dexamethasone alone in patients with newly diagnosed myeloma without intent for immediate autologous stem cell transplant (SWOG S0777): a randomized, open-label, phase 3 trial. Lancet 2017;389:519-527.
2. Attal M, Lauwers-Cances V, Hulin C, et al. Autologous transplantation for multiple myeloma in the era of new drugs: a phase III study of the Intergroupe Francophone Du Myelome (IFM/DFCI 2009 trial) [abstract] Blood 2015;126:Abstract 391.

3. Attal M, Palumbo A, Holstein SA, et al. Lenalidomide (LEN) maintenance (MNTC) after high-dose melphalan and autologous stem cell transplant (ASCT) in multiple myeloma (MM): a meta-analysis (MA) of overall survival (OS) [abstract]. J Clin Oncol 2016;34(Suppl):Abstract 8001.

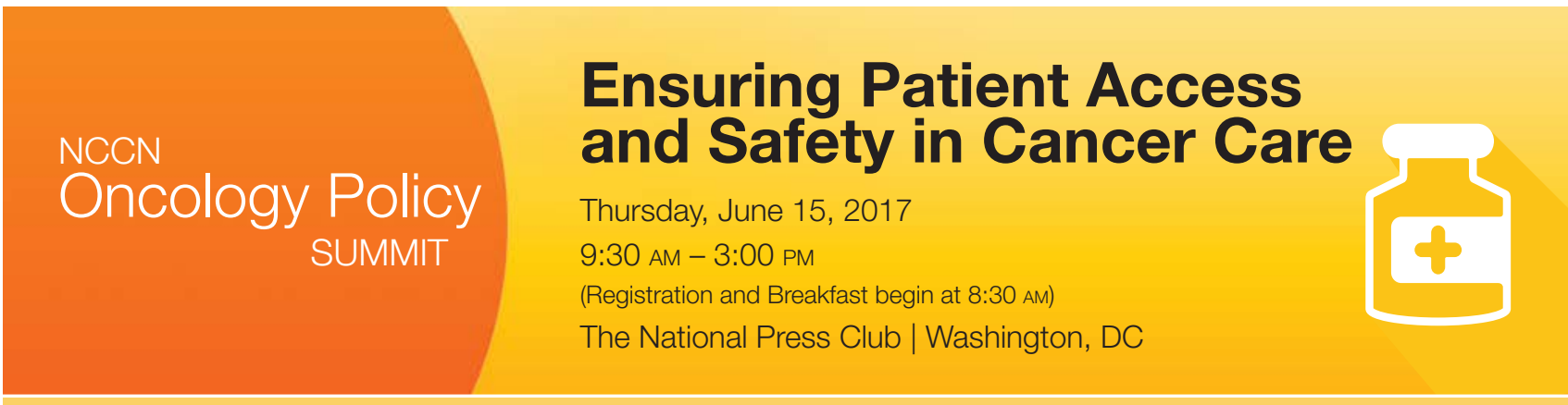

This summit will provide an opportunity for a varied group of stakeholders including providers, payers, patients, patient advocacy organizations, and industry, to thoughtfully discuss patient access to high-quality, effective, and efficient oncology care, as well as to disseminate advances in oncology patient safety systems best practices, processes, and policies. The summit will also explore shifting health care priorities under the Trump Administration and any implications for safety and access to high quality cancer care.
Agenda* topics include:

- Safety and Accountability in Oncology Care

- Patient Access to Safe, High Quality Cancer Care Under a New Administration

- Panel Discussions:

O Safety and Accountability in Cancer Care: Past, Present, and Future

○ Barriers and Opportunities in Ensuring Access to Safe, High Quality Cancer Care

${ }^{*}$ Agenda subject to change

\section{NCCN.org/policy}

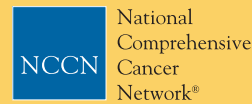

Supporters

Supported by Adaptive Biotechnologies; AmerisourceBergen; Apobiologix, a division of ApoPharma USA, Inc.; EMD Serono; Gilead Sciences, Inc. HELSINN; Incyte Corporation: Janssen Oncology: National Decision Support Company; and Pharmacyclics UC, An AbbVie Company. Sponsored by AstraZeneca; Boehringer Ingelheim Pharmaceuticals, Inc.; Boston Biomedical, Inc.; and Dendreon. This activity is supported by a contribution from Lilly. Supported by an independent educational grant from Merck \& Co., Inc. Supported by an unrestricted sponsorship from TESARO. (As of April 11, 2017) 